
internationales

vol. 30 - n² | 2014

Composer (avec) la frontière. Passages, parcours migratoires et échanges sociaux

\title{
Schor Ralph, Écrire en exil. Les écrivains étrangers en
}

France, 1919-1939

\section{Yves Charbit}

\section{(2) OpenEdition}

\section{Journals}

Édition électronique

URL : https://journals.openedition.org/remi/6827

DOI : $10.4000 /$ remi.6827

ISSN : $1777-5418$

Éditeur

Université de Poitiers

Édition imprimée

Date de publication : 1 juin 2014

Pagination : 195-197

ISBN : 979-10-90426-22-1

ISSN : 0765-0752

Référence électronique

Yves Charbit, « Schor Ralph, Écrire en exil. Les écrivains étrangers en France, 1919-1939 », Revue

européenne des migrations internationales [En ligne], vol. 30 - n² | 2014, mis en ligne le 01 juin 2014,

consulté le 14 avril 2022. URL : http://journals.openedition.org/remi/6827 ; DOI : https://doi.org/

$10.4000 /$ remi.6827 


\section{Notes de lecture}

Vora, Neha

Impossible Citizens: Dubai's Indian

Diaspora. - Durham \& London: Duke

University Press, 2013. -264 p.

ISBN : 978-0-82-235378-2 ; 978-0-82-25393-5

Le Golfe arabique, troisième pôle migratoire mondial, est un terrain d'étude en extension, pour les spécialistes des migrations (Gardner, 2005 ; Ali, 2010 ; Kamrava et Babar, 2012 ; Mahdavi, 2011) comme pour ceux des études urbaines (Elsheshtawy, 2010 ; Kanna, 2011). Neha Vora pour sa part s'intéresse aux modes d'appartenance des migrants dans le contexte de l'État rentier autoritaire de Dubaï. Ici, la gouvernementalité crée, grâce à des structures rigides de citoyenneté et de migration, des " outsiders " et des " sujets néolibéraux " (p. 21). L'identité nationale émiratie se construit en effet par opposition aux étrangers qui forment plus de $80 \%$ de la population, mais sont exclus des formes politiques de citoyenneté et de l'état providence. L'auteur s'appuie à la fois sur Ong et Agamben pour montrer que l'exception (ici l'exclusion des étrangers de la citoyenneté) participe à la souveraineté de l'État émirati. Bien que la présence des Indiens soit un fait avéré de longue date, que Dubaï et l'Inde soient liés par le commerce depuis des siècles, les Émirats Arabes Unis cherchent à effacer l'ancien cosmopolitisme précolonial et colonial pour bâtir une identité arabe expurgée et " pure " (p. 48). La dichotomie avant/après le pétrole est utilisée pour montrer que la présence des étrangers dans le pays est un mal nécessaire, mais foncièrement temporaire. Or de nombreux Sud-asiatiques sont installés à Dubaï depuis des décennies. Cette communauté est à la fois « transitoire et établie " (p. 66), invisible entre les travailleurs exploités dont l'exposition médiatique croit, et l'élite internationale, image de marque de Dubaï. L'auteur pose donc la question de l'appartenance (belonging) de la classe moyenne indienne dont le sentiment de déchéance et de non appartenance à l'émirat ne fait pourtant pas se tourner vers la diaspora indienne.

Les commerçants représentent ces " citoyens non officiels " (p. 93) qui auraient néanmoins un poids politique important grâce à l'ancienneté de leurs réseaux et au fait qu'ils sont des acteurs incontournables du système de la kafala. Ils produisent de l'autorité étatique et des hiérarchies parmi les Sud-asiatiques. Sans revendications politiques, ils se reconnaissent dans la liberté économique émiratie et l'entreprenariat. Ils sont des individus représentatifs de l'ordre néolibéral pour qui la citoyenneté passe par la consommation (p. 135). II en va autrement pour les jeunes Sud-asiatiques nés à Dubaï qui se forgent peu à peu une "citoyenneté diasporique " (p. 148). En entrant dans I'une des universités occidentales implantées à Dubaï, ils passent de la société indienne fermée sur elle-même, aux écoles communautaires, à celle qu'ils pensent plus ouverte, aux universités formant "à des modalités néolibérales de citoyenneté globales " (p. 159). Mais ils vivent alors la division citoyens/non-citoyens dans leur chair par des expériences de discriminations et de racisme. Leurs aspirations au changement à Dubaï sont politiques, mais vaines. Comme leurs parents, ils se vivent comme "Indiens par défaut " (p. 149), car Dubaï, l'autre base de leur identité, ne leur donne le choix que d'une appartenance minimale, celle de la " citoyenneté urbaine " (p. 153). L'entrée à l'université s'accompagne donc de désillusions et de la prise de conscience de n'être que des " citoyens de deuxième classe " (p. 163). Néanmoins, l'Inde est rarement envisagée comme une solution pour la mobilité alors que l'Occident attire. 
Avec le développement du New Dubaï, son statut de marque commerciale internationale et son nouveau cosmopolitisme global, la classe moyenne indienne, représentant l'ancien cosmopolitisme, a du mal à se situer. Ses quartiers de vie, tel Bur Dubaï où l'auteur a conduit une partie de ses enquêtes, sont exclus des projets urbains d'une ville de plus en plus ségréguée. Le vieux Dubaï, qui n'est pas un " ailleurs" (p. 66) pour les Indiens, mais bien une " extension de l'Inde " (p. 66) est invisible pour le gouvernement, les touristes et les expatriés. Du coup, les Indiens, qui ont le sentiment d'avoir construit Dubaï, se sentent marginalisés. L'insécurité liée à leur statut temporaire, la non reconnaissance de droits quelconques et la nouvelle globalisation les rendent vulnérables. Pourtant, dans un contexte où la citoyenneté par la consommation ne semble qu'un pis-aller, la diaspora indienne n'est pas une identité mobilisée, I'Inde n'est pas une " maison " (p. 84), aucune nostalgie ne s'exprime vis-à-vis du pays de leurs ancêtres. Les discours des Indiens " révèlent qu'ils sont à la limite (liminal) de la diaspora indienne, de la nation indienne et de la vision du futur du gouvernement de Dubaï » (p. 89). Dubaï reste le lieu principal d'appartenance de ces populations, qui ne sont pas attachées à I'Inde autrement que par le biais d'une culture réifiée. La classe moyenne indienne à Dubaï ne serait donc pas à proprement parler diasporique, à la fois rejetée par I'Inde et par Dubaï.

Dès l'introduction, l'auteur prend le parti pris théorique de ne pas « exceptionaliser " ni " pathologiser " Dubaï (p. 12) en affirmant que les formes de gouvernance et de citoyenneté sont multiples et circulent dans le monde. En suivant Ong (1999), elle souhaite se démarquer des conceptions occidentales de la citoyenneté, pour montrer que dans le cadre de la globalisation, la citoyenneté ne repose pas que sur l'appartenance à un État-nation, mais qu'elle peut s'exprimer par la consommation, I'urbanité ou la diaspora. Sa volonté démonstrative se heurte néanmoins à son terrain (les jeunes Indiens aimeraient des droits politiques à Dubaii) et au fait qu'elle confonde souvent appartenance (à la société libérale, à la sphère économique) et citoyenneté au sens de droits politiques fondamentaux (droit de vote, liberté d'association, etc.). Finalement son désir initial de banaliser Dubaï n'est pas conforté par son développement où l'on voit que les " citoyens impossibles " le restent malgré une " citoyenneté urbaine " et que l'entredeux n'est pas une situation confortable et mène à une impasse identitaire. La classe moyenne indienne serait donc typique du néo-libéralisme qui ne donne de l'appartenance que par la position économique et la liberté de choisir (un emploi, une consommation), mais refuse les droits politiques.

Malgré son intérêt et son approche novatrice, l'ouvrage de Neha Vora souffre d'une empirie déficiente. Cela révèle les limites d'un cadre théorique fort imposé dès le départ et quasiment prédéterminant de l'analyse. Rien n'est dit sur la méthodologie ni les personnes rencontrées, un éventuel échantillon ou les méthodes d'enquêtes. Les interprétations de l'auteur sont peu agrémentées de témoignages, ce qui est surprenant pour une étude anthropologique. Aucune proposition ou témoignage ne vient contredire des assertions cadrées dans l'introduction. II en résulte une confusion dans les catégories étudiées, qui ne sont jamais vraiment définies ou discutées. Les marchands d'or au train de vie luxueux étudiés dans le chapitre 2 font-ils vraiment partie de la classe moyenne indienne ? Qui sont les parents des étudiants sud-asiatiques qui déboursent plusieurs milliers de dollars pour s'inscrire dans une université occidentale ? Les catégories South Asian, Indian, sont-elles vraiment interchangeables ? Pourquoi la mobilité sociale est-elle si peu étudiée ? Autant de questions sans réponse qui ne viennent cependant pas réduire l'intérêt de ce livre qui le premier propose une étude d'un groupe de population peu étudié, mais central à la vie économique du Golfe. 


\section{..? Références bibliographiques}

Ali Syed (2010) Dubai: Gilded Cage, New Haven, Yale University Press, 360 p.

Elsheshtawy Yasser (2010) Dubai: Behind an Urban Spectacle (Planning, History and Environment Series), NewYork, Routledge, $304 \mathrm{p}$.

Gardner Andrew M. (2005) City of Strangers: The Transnational Indian Community in Manama, Bahrain, PhD Dissertation, Department of Anthropology, University of Arizona.

Kamrava Mehran and Babar Zahra (2012) Migrant Labor in the Persian Gulf, New York, Columbia University Press, 276 p.

Kanna Ahmed (2011) Dubai, the City as Corporation, Minneapolis, University of Minnesota Press, 296 p.

Mahdavi Pardis (2011) Gridlock: Labor, Migration and Human Trafficking in Dubai, Stanford, Stanford University Press, 264 p.

Ong Aihwa (1999) Flexible Citizenship: The Cultural Logics of Transnationality, Durham, Duke University Press, 322 p.

Tristan Bruslé

CNRS

Centre d'Études Himalayennes

\section{Schor, Ralph}

Écrire en exil. Les écrivains étrangers en France, 1919-1939. - Paris : CNRS Éditions, 2013. $-342 \mathrm{p}$

ISBN : 978-2-271-07624-3

Le livre passionnant que vient de publier Ralph Schor aux éditions du CNRS nous offre en 342 pages une remarquable peinture, fine et fouillée, de la situation des écrivains étrangers en France entre 1919 et 1939. L'ouvrage est fondé sur un solide corpus de 311 écrivains de vingtdeux nationalités, principalement des Allemands, des Russes et des Américains. On trouvera en annexe de très utiles notices biographiques, suivies d'une riche bibliographie bien organisée. Sont d'abord présentées les sources primaires, c'est-à- dire la propre production des écrivains : mémoires d'orientation autobiographique, correspondance et leurs œuvres (romans, essais, articles).

Le premier chapitre marque d'emblée la dualité fondamentale de ces situations d'exil. D'une part, l'exil volontaire caractérise surtout les Américains (mais pas seulement eux) ; il s'agit d'une fuite loin du conformisme parfois étouffant des pays d'origine. D'autre part, un exil forcé a été induit par l'oppression politique ou raciale. Derrière cette première grande opposition, la réalité est plus complexe. Ainsi, le groupe des Américains de " la génération perdue " n'est guère homogène, tant s'en faut. II n'y a probablement rien de commun, sinon l'exil, entre la pauvreté d'Henri Miller, le dandysme de Scott Fitzgerald, riche et désoeuvré, les convictions communistes de I'Afro-Américain Claude McKay, ou encore l'affichage par Gertrude Stein et Nathalie Barney de leur homosexualité, qu'elles peuvent vivre librement à Paris. Fuir un milieu étouffant et conservateur est aussi la motivation de certains Européens, tels le Belge Robert Kanters ou I'Irlandais Samuel Becket. L'exil forcé caractérise bien sûr les exilés allemands, à partir de l'arrivée au pouvoir d'Hitler en 1933. Comme l'écrit Klaus Mann, le fils du prix Nobel Thomas Mann, rester en Allemagne offrait un choix impossible " entre le martyr absurde et la trahison opportuniste, le camp de concentration ou l'intégration " (p. 25). Les dictatures fascistes d'Europe centrale, d'Espagne et du Portugal, et à l'autre bout du spectre politique la révolution bolchevique, contraignirent à l'exil intellectuels, artistes ou militants politiques.

Le deuxième chapitre de l'ouvrage reprend le proverbe allemand et yiddish "Heureux comme Dieu en France ", que I'on associe en général à la France des Droits de l'homme et du citoyen et plus généralement à I'héritage des Lumières. Ce que Ralph Schor souligne, c'est plutôt I'attirance exercée sur les intellectuels et les artistes par le cosmopolitisme de 
la France des années 1920 et 1930, la liberté d'expression, le rayonnement culturel, le bouillonnement intellectuel qui y règnent. Les grands noms abondent, qui témoignent de la puissance d'attraction de la France, même si certains n'y passèrent que quelques années avant de s'exiler à nouveau. Pour d'autres, un enracinement définitif a permis l'épanouissement de leur talent, comme le montre le superbe chapitre 7 intitulé Exil et Littérature. Ce sont par exemple Arthur Adamov, Hanah Arendt, Victor Bash, Walter Benjamin, Nina Berberova, Marthe Bibesco, Louis Bromfield, Walter Benjamin, Émile Cioran, Alfred Doblin, John Dos Passos, Lion Feuchtwanger, Romain Gary, Peggy Guggenheim, Ernest Hewingway, Eugène lonesco, James Joyce, Arthur Koestler, Maurice Maeterlink, Heinrich Mann, Man Ray, Ludwig Marcuse, Somerset Maugham, Irène Némirovsky, Anna de Noailles, Joseph Roth, Nathalie Sarraute, Georges Simenon, Elsa Triolet, Tristan Tsara. En 1953, dans Souvenirs, souvenirs, Henri Miller note : "À Paris, on n'a pas besoin de stimulants artificiels pour créer. L'atmosphère est saturée de création ". Leur inspiration s'enrichit certes des auteurs du XIXe siècle, Balzac, Stendhal, Hugo, Flaubert ou Zola, mais aussi de leurs contemporains : Marcel Proust, Anatole France, Romain Rolland, Paul Valéry, André Gide surtout, font autorité. À côté des qualités littéraires et des modèles que constituent des romans tels que Le Rouge et le Noir, Madame Bovary ou À la recherche du temps perdu, " la fréquentation des auteurs français du XXe siècle [observe Ralph Schor] conduisait à deux obligations intellectuelles et morales pressantes : l'attachement aux valeurs humanistes et le nécessaire engagement de l'écrivain dans les combats contemporains "(p. 172). Cela est sans doute vrai de toute grande littérature, mais la tolérance et l'ouverture furent spécifiques de la société française des années 1920 et 1930 et elles eurent une influence directe et profonde sur le processus créatif des écrivains en exil (on pourrait d'ailleurs en dire de même du monde des peintres et des sculpteurs). Henri Miller mérite à nouveau d'être cité. II décrit l'écriture du Tropique du Cancer comme un acte désespéré. "Désespoir, fureur, récrimination, violence. Je voulais tout mettre à sac, tout. Oh oui, vraiment, mon écriture a subi un changement définitif. Je ne sais pas exactement comment expliquer cela. Sauf en disant que je vivais dans une atmosphère de complète liberté et d'anarchie " (p. 174). Même témoignage d'Ernest Hemingway, qui considérait qu'il avait appris à écrire à Paris. Mais qu'est-ce qu'une littérature d'exil ? Les Russes en particulier furent confrontés à la difficulté de se couper de leurs racines, au risque de s'enfermer dans une nostalgie stérile. D'autres au contraire puisèrent dans la littérature d'avant-garde, Joyce, Céline, Proust, le renouvellement de leur inspiration tout en nourrissant leurs œuvres de leur identité même, de leurs propres souvenirs ou de leurs travaux critiques d'histoire littéraire (sur les vies de Tourgueniev, Tolstoï ou Pouchkine). Enfin, le roman historique permit aux Allemands une critique indirecte du nazisme à travers des personnages sanguinaires de l'histoire romaine tels Néron.

Le livre est également une contribution très importante à l'articulation entre Exil et politique (chapitre 8), dimension évidemment déterminante de la présence en France de ces écrivains, d'autant qu'après la tragédie de la Grande Guerre, I'insouciance des années 1920 fit progressivement place à un assombrissement croissant : d'abord la montée du fascisme italien (1922), puis la crise économique (1929), le nazisme (1933) et enfin la guerre civile espagnole (1936), véritable répétition générale avant l'embrasement de l'Europe en 1939. Ralph Schor évoque bien sûr l'engagement antifasciste et présente avec finesse la palette des positionnements et des stratégies. Si les intellectuels et les artistes européens furent les témoins douloureux de ces événements majeurs, le cas particulier des Russes doit être évoqué, car après la révo- 
lution de 1917, la guerre civile en Russie et le triomphe des bolcheviks déclenchèrent l'exode des Russes blancs. La réalité ne saurait pourtant se réduire à l'exil des seuls vaincus. On trouvait en France à la fois le dernier carré des monarchistes qui souhaitaient restaurer le tsarisme, mais aussi les libéraux favorables à une monarchie constitutionnelle ou encore des démocrates républicains, sans compter les différentes appartenances religieuses et enfin les différences entre les générations. Les appartenances politiques et les choix idéologiques, qui transcendaient les nationalités, compliquent encore le paysage de l'exil politique en France. On pense bien sûr aux communistes et aux manipulations internationales de Staline surtout lorsque se met en place la répression contre les trotskistes.

Ce qui fait aussi l'intérêt du livre c'est la richesse des analyses du rapport de ces exilés à la France (chapitres 2, 3 et 6). La représentation de la France qu'ils portaient en eux avant l'exil, c'est évidemment celle d'une terre de culture et de modernité, d'un pays doté d'une capitale unique au monde, bénéficiant aussi d'une riche diversité régionale. La culture française est pour eux celle de la raison et de la mesure, ce qui n'exclut nullement la passion des Français pour les débats d'idées et l'importance des valeurs humanistes. Bref un art de vivre envié par toute I'Europe. Mais l'expérience de l'exil, c'est aussi le racisme et la xénophobie, en tout cas la méfiance et l'isolement, même si de très nombreuses occasions et lieux de rencontre permettent de limiter la solitude: dans de nombreux salons, salles de conférence, cafés et maisons d'édition circulent les idées, les informations, les rares pistes d'emploi. Sauf pour la poignée d'Américains dotés d'une grande fortune personnelle, la plupart des exilés connaissent des conditions de vie sommaires, dans des appartements glacés I'hiver, souvent miteux et insalubres. La précarité, la faim, le froid, le déclassement social est leur lot quotidien, en dépit d'une incon- testable solidarité (chapitre 4). Aussi se produit, comme pour toute immigration, un réajustement en baisse des attentes et des représentations, ce qui ne manque pas de déclencher chez certains une crispation identitaire. La souffrance est constante chez la poétesse Marina Tsvetaeva, incandescente et fermée à tout compromis, dont Zoé Oldenburg écrivit qu'elle « vivait séparée du monde entier par la terrible musique des mots qui la brûlaient jour et nuit » (p. 184).

En fin de compte, le titre Écrire en exil est parfaitement choisi. Le livre illustre de manière très convaincante la nécessité d'une contextualisation fine de la production littéraire. En ce sens il s'inscrit dans la tradition de la sociologie de la connaissance, non pas de manière schématique en postulant que toute production littéraire n'est que le produit d'un strict déterminisme, mais en montrant comment les conditions concrètes de l'exil dans le contexte spécifique de la France, ont influé sur la production littéraire. II s'agit d'un grand livre, qui fera référence, remarquablement écrit, nourri d'une vaste culture et qui se situe au confluent de deux champs de spécialité de l'auteur. Ces quelques lignes ne pouvaient prétendre en restituer toute la richesse.

Yves Charbit CEPED

Université Paris Descartes-INED-IRD

Delcroix, Catherine

Ombres et lumières de la famille Nour.

Comment certains résistent face à la

précarité (nouvelle édition). - Paris : Petite

Bibliothèque Payot, 2013. - 302 p.

ISBN : 978-2-228-90849-8

Préfacé par Françoise Lorcerie et augmenté d'une nouvelle postface de l'auteur, sorte d'épilogue faisant le bilan des aventures post-édition initiale (2001), heureuses ou souvent malheureuses des membres de la famille Nour, ce petit 
ouvrage qui en est à sa troisième édition $(2001,2005,2013)$ nous raconte la vie quotidienne d'une famille immigrée d'origine marocaine en France, évidemment sous un nom d'emprunt et sur un site non précisé, emblématique de ce que le jargon médiatico-politique nomme les "quartiers ", par ce curieux glissement de sens qui caractérise beaucoup de faits socio-politiques. L'auteure, sociologue française d'origine bruxelloise, a réussi, comme le souligne Françoise Lorcerie, à écrire une sorte de " roman vrai " qui par certains côtés mériterait d'être transcrit en scénario cinématographique. La famille "Nour ", pseudopatronyme signifiant " lumière " en arabe, rappelle également Françoise Lorcerie, est réellement emblématique d'une réalité quotidienne que l'on peut côtoyer tous les jours et partout, non seulement en France, mais probablement dans une très grande partie de l'Europe post-industrielle; elle pourrait être d'origine turque en Allemagne ou en Suède, pakistanaise ou bengladeshie en Grande-Bretagne, mais pourquoi pas mexicaine ou portoricaine aux États-Unis, haïtienne ou marocaine au Canada. Les variables nationales (au sens de pays d'origine) ou religieuses (ici, musulmanes) peuvent être mobilisées par certains des membres de la famille suivie on pourrait presque ici parler de cohorte dans la mesure où le travail s'est poursuivi sur plus de quinze années -, peut-être plus encore par l'extérieur, mais au final elles n'apparaissent jamais essentielles. II s'agit bien ici d'une famille rurale, oscillant entre conservatisme traditionnel de la communauté du village de montagne marocain et découverte de la modernité citadine européenne, qui, tant bien que mal, tente, au prix de grandes souffrances et de difficultés incessantes de s'intégrer dans une société urbaine occidentale. On pourrait reprocher à l'auteure de recourir aux poncifs de I'intégration: Djamila, la mère courageuse, et Leilla, la fille travailleuse et volontaire, s'opposent aux personnages falots ou déviants que sont Amin le père, Rachid, Djamel, Driss et Toufik. Toufik, le petit dernier et Fatiha, la petite sœur (qui elle aussi travaille bien à l'école), se trouvent en position intermédiaire. Or les garçons sont pris entre leur rôle traditionnel de garçon dans une société patriarcale et leur situation d'enfants d'immigrés dans une société démocratique, mais souvent plus de façade dès lors que l'on est immigré ou réfugié, en tous cas d'origine étrangère. Or mère et filles sont prises dans leur rôle féminin de transmission de la stabilité, de la mesure en toute chose. La notion d'intégration est ici sévèrement mise à mal par le vécu quotidien de personnages dont le plus grand défaut est sans doute leur désespérante " normalité ". Ils ne sont ni exceptionnels, ni héroïques, ni malfaisants, ils essaient tout simplement de vivre décemment et parfois de s'autoriser à rêver à un avenir meilleur, la raison essentielle de la présence en France ou en Europe de leurs parents !

Le bilan de l'auteure, et peut-être plus encore son épilogue, laisse au lecteur un sentiment étrange, celui d'un gâchis assez scandaleux, touchant des personnes modestes, et plus encore leurs enfants dont le défaut principal est d'être d'origine maghrébine dans une société française qui n'est pas celle qu'elle prétend être en matière d'intégration. Les acteurs positifs ne sont pourtant pas absents : enseignante, travailleur social, simple voisin-e de palier, etc., mais leur action, qui peut être décisive sur un dossier, à un moment, ne suffit pas à gommer ce sentiment dérangeant. La famille Nour est sans aucun doute emblématique de beaucoup de familles immigrées et de leurs descendants, en France ou ailleurs ; ses membres finiront probablement par réellement s'intégrer économiquement et socialement, mais à quel prix, avec quels dommages collatéraux?

Stéphane de Tapia CNRS Université de Strasbourg 